\title{
Antialgal Synergistic Polystyrene Blended with Polyethylene Glycol and Silver Sulfadiazine for Healthcare Applications
}

\author{
M. Raheel Anjum $\mathbb{D}^{1},{ }^{1}$ Shehla Mushtaq ${ }^{D},{ }^{2}$ M. Asad Abbas $\mathbb{D}^{1}$, Azhar Mahmood $\mathbb{D}{ }^{2}$ \\ Habib Nasir $\mathbb{D}^{2}{ }^{2}$ Hussnain A. Janjua $\mathbb{D}^{3},{ }^{3}$ Qamar Malik, ${ }^{4}$ and Nasir M. Ahmad ${ }^{1}{ }^{1}$ \\ ${ }^{1}$ Polymer Research Lab, School of Chemical and Material Engineering (SCME), National University of Sciences and \\ Technology (NUST), Sector H-12, Islamabad 44000, Pakistan \\ ${ }^{2}$ Department of Chemistry, School of Natural Sciences (SNS), NUST, Sector H-12, Islamabad 44000, Pakistan \\ ${ }^{3}$ Department of Industrial Biotechnology, Atta-Ur-Rahman School of Applied Biosciences (ASAB), NUST, Sector H-12, \\ Islamabad 44000, Pakistan \\ ${ }^{4}$ ABBOTT Energy \& Environment Inc., Alastair Ross Technology Centre, 3553 31st Street NW, Calgary, AB, Canada T2L 2 A6 \\ Correspondence should be addressed to Nasir M. Ahmad; nasir.ahmad@scme.nust.edu.pk
}

Received 21 October 2020; Revised 3 October 2021; Accepted 22 October 2021; Published 25 November 2021

Academic Editor: Kinga Pielichowska

Copyright (c) 2021 M. Raheel Anjum et al. This is an open access article distributed under the Creative Commons Attribution License, which permits unrestricted use, distribution, and reproduction in any medium, provided the original work is properly cited.

\begin{abstract}
Polystyrene (PS) was blended with polyethylene glycol (PEG) and silver sulfadiazine (SS) with different weight proportions to form polymeric blends. These synthesized blends were preliminary characterized in terms of functional groups through the FTIR technique. All compositions were subjected to thermogravimetric analysis for studying thermal transition and were founded thermally stable even at $280^{\circ} \mathrm{C}$. The zeta potential and average diameter of algal strains of Dictyosphaerium sp. (DHM1), Dictyosphaerium sp. (DHM2), and Pectinodesmus sp. (PHM3) were measured to be $-32.7 \mathrm{mV},-33.0 \mathrm{mV}$, and $-25.7 \mathrm{mV}$ and $179.6 \mathrm{~nm}, 102.6 \mathrm{~nm}$, and $70.4 \mathrm{~nm}$, respectively. Upon incorporation of PEG and SS into PS blends, contact angles were decreased while hydrophilicity and surface energy were increased. However, increase of surface energy did not led to decrease of antialgal activities. This has indicated that biofilm adhesion is not a major antialgal factor in these blended materials. The synergetic effect of PEG and SS in PS blends has exhibited significant antialgal activity via the agar disk diffusion method. The PSPS10 composition with $10 w / w \%$ PEG and $10 w / w \%$ SS has exhibited highest inhibition zones $10.8 \mathrm{~mm}, 10.8 \mathrm{~mm}$, and $11.3 \mathrm{~mm}$ against algal strains DHM1, DHM2, and DHM3, respectively. This thermally stable polystyrene blends with improved antialgal properties have potential for a wide range of applications including marine coatings.
\end{abstract}

\section{Introduction}

Biofouling is an undesired build-up of biotic deposits on a material's surface. This biotic deposit includes different species such as cyanobacteria, algal spores, fungi, and macroalgae [1]. Biofouling thus may be either microbial based or combination of micro- and macroattack caused by the synergetic effect of microbial and macroorganism, respectively [2] . Algal biofouling is a specific type of biodegradation initiated by algae in highly humid as well as aquatic environment and multiplies in their numbers that lead to attachment of other biological species spiraling it into complex heterogeneous system of macrobiofouling [1]. The biological degradation of certain material depends specifically on the type of microorganisms, environmental conditions, and its physical and chemical properties [3]. Microbial growth adversely affects numerous areas relevant to food preservation, water purification, photobioreactors, bioimplants, and biosensors in healthcare applications [4]. Microbial growth can be inhibited by different types of materials like nanocomposite, polymer composites, and polymeric systems [5]. Among these materials, polymer-based systems are the best antimicrobial materials and have numerous advantages over conventional materials with exceptional film-forming ability, suitable chemical activity, thermal stability, mechanical strength, corrosion resistance, and low cost [6]. Various antifouling 




FIGURE 1: Schematic of various blends showing antialgal properties.

polymer systems were developed to exhibit increased efficiency, enhanced antimicrobial behavior, process ability, tunable properties, low toxicity, prolonged life time, and diverse functionalities [7, 8]. Therefore, antifouling polymer-based systems have given an innovative direction to the research in the field of antimicrobial materials. Surface features like hydrophilicity, surface roughness, polarity, surface energy, and contact angle are important parameters to design antifouling systems [9]. Polymer-based antifouling blends are used in a wide variety of forms including copolymers, grafting, polymer coatings, thin films, polymer brushes, and polyelectrolytes [10].

Gitchaiwat et al. have enhanced antialgal property of LDPE against the Chlorella vulgaris TISTR 8580 species by adding the antialgal agent such as 2-methylthio-4-ethylamino-6-tert-butylamino-triazin-1,3,5 [terbutryn (TT)] [11]. The results have shown that TT with an optimal loading of $750 \mathrm{ppm}$ could be used to effectively inhibit the growth of C. vulgaris, but to get satisfactory inhabitation iodopropynyl butylcarbamate (IPBC) could be used as antialgal promoters in the LLDPE specimens [11]. In another study, Yandi and coworkers have investigated the antialgal activity of cationic poly(2-(dimethylamino)ethyl methacrylate) (PDMAEMA) brushes against marine algae Ulva linza and U. lactuca [12]. PDMAEMA has been found to destroy zoospores in contact with it and also avoid the subsequent development of normally settled spores [12]. Bodkhe et al. have used PSBMA as part of a block copolymer with PDMS that was incorporated into the polyurethane network. Although these coatings had excellent fouling resistance properties against the marine bacterium Halomonas pacifica and N. incerta, the performance was less pronounced against algal species Cellulophaga lytica and U. linza [13]. In a recent study, 3sulfopropylmethacrylate was copolymerized with methyl methacrylate that only controls the adhesion of Dictyosphaerium algae but did not kill the algal cells [14]. These literature studies have indicated that the most polymeric systems could significantly inhibit bacterial species but could not be used effectively against algal species. To overcome this discrepancy, hydrophilic polymers such as PEG could be introduced to the polymeric system [15]. PEG due to polar nature and strong hydrogen bonding leads to excluded volume which makes the adsorption of fouling organisms extremely difficult on its surface [16]. PEG-based antifouling system acts as a biopassive material which effectively reduces the microbial adhesion owing to the formation of an interfacial layer [17]. The PEG moieties present on various frictional surfaces have shown strong resistance towards nonspecific proteins and cell adsorption. This minimizes the amount of bioadhesion on these surfaces and makes PEG highly desirable for antifouling purposes [17, 18].

In the current novel work, blending of polystyrene (PS), polyethylene glycol (PEG), and silver sulfadiazine was carried out by varying the concentration of constituents via the melt-blending technique. Blend synthesis was confirmed through FTIR, and thermal stability was studied through TGA. The equilibrium contact angle of various compositions was measured, and the surface energy of compositions was calculated with the help of contact angle. The antialgal assay of all blends was performed against three different algal strains Dictyosphaerium sp. (DHM1), Dictyosphaerium sp. (DHM2), and Pectinodesmus sp. (PHM3) by the agar disk diffusion method via zone of inhibition measurement. The PSPS10 blend has demonstrated a maximum zone of inhibition against these algal strains. Figure 1 shows the antialgal behavior of the various blends indicating PSPS10 has the maximum antialgal efficiency.

\section{Experimental}

2.1. Materials. All the chemical and reagent are of analytical grade. Polyethylene glycol (Mw-1000, 99.99\%) was purchased from Daejung Chemicals, S. Korea, Polystyrene (Melt flow index 2-4 g/10 min; Mw-350000, 95\%, average 
TABLe 1: Compositions of blends.

\begin{tabular}{lccc}
\hline Sample code & PS $w / w \%$ & PEG $w / w \%$ & SS $w / w \%$ \\
\hline PS & 100 & 0 & - \\
PSP5 & 95 & 5 & - \\
PSP10 & 90 & 10 & - \\
PSP15 & 85 & 15 & - \\
PSP20 & 80 & 20 & - \\
PSS10 & 90 & - & 10 \\
PSPS10 & 80 & 10 & 10 \\
\hline
\end{tabular}

Mn 170,000, density $1.04 \mathrm{~g} / \mathrm{cm} 3$ ), and silver sulfadiazine (98\%) was purchased from Sigma Aldrich, Germany. Mueller Hinton Agarose was purchased from Merck, UK. Standard algal strains Dictyosphaerium sp. (DHM1), Dictyosphaerium sp. (DHM2), and Pectinodesmus sp. (PHM3) were used as testing species.

2.2. Preparation of Blends. The melt-mixing technique was used for the preparation blends of polystyrene (PS) with polyethylene glycol (PEG), and silver sulfadiazine (SS) was prepared using the internal batch mixer of HAAKE Rheomix model Lab OS by Thermo Fisher Scientific, USA. PEG and SS were utilized as received while PS was meshed to fine powder prior to blend preparation. The temperature of the equipment was raised up to $200^{\circ} \mathrm{C}$ and held for five minutes to become stabilized on attaining the desired temperature. Afterward, the predetermined composition of blends was fed into the melt mixer, and its mixing was controlled by optimizing parameters such as RPM, temperature, and time. After completion of blending, the blends were retrieved and further molded into sheets of $1 \mathrm{~mm}$ thickness by using laboratory press by Gibitre Instrument, Italy. The prepared sheets were finally cold pressed using 30 -ton hydraulic press. Table 1 shows blend composition that were made by varying the amount of PEG from 0 to $20 \%(w / w)$ and SS from 0 to $10 \%(w / w)$.

2.3. Characterization. Infrared spectroscopy (ATR-FTIR) was performed by Bruker's model Alpha-P (USA) for identification of functional groups in various blend compositions. Thermal degradation analysis was performed using TGA model LF2 (Mettler Toledo, USA) equipped with high-temperature furnace in nitrogen atmosphere at the heating rate of $20^{\circ} \mathrm{C} / \mathrm{min}$. The contact angle of blends was measured by using $5 \mu \mathrm{L}$ sessile drop of deionized water via a lab-made customized apparatus. The contact angle was measured for each composition by average three values of drops after 2 min with the help of ImageJ software. Since the surface energy of the substrate is based on the contact angle measurement of blends, therefore, the surface energy was calculated by the Chibowski equation [14].

$$
\gamma s=\frac{\gamma l}{2}\left(1+\cos \theta_{E q}\right)
$$

Here, $\gamma s$ is the solid surface energy, $\gamma l$ is the surface energy of liquid, and $\theta_{E q}$ is water equilibrium contact angle.

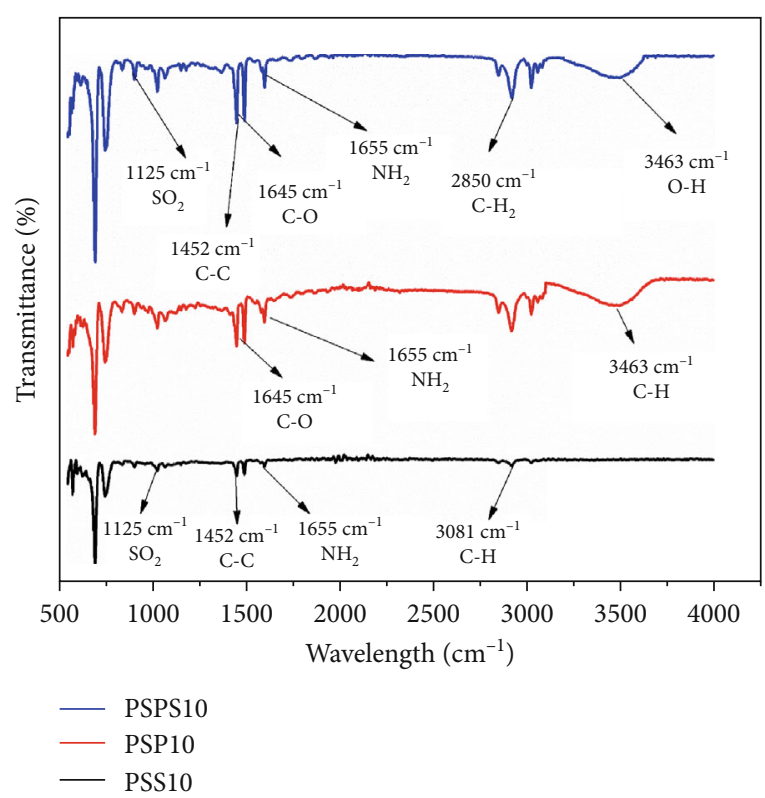

FIGURE 2: FTIR spectra of PSPS10, PSP10, and PSS10.

The zeta potential and zeta size of algal strains Dictyosphaerium sp. (DHM1), Dictyosphaerium sp. (DHM2), and Pectinodesmus sp. (PHM3) were measured using the Zetasizer model Nano Zsp by Malvern Instruments, UK. Prior to zeta potential measurement, cultures were kept undisturbed for almost 10 minutes to let any flocks settle down, and strains were sampled from the top to be used in experiment.

2.3.1. Antialgal Bioassay. The antialgal profile of various polymeric blends was tested against three different algal strains namely Dictyosphaerium sp. (DHM1), Dictyosphaerium sp. (DHM2), and Pectinodesmus sp. (PHM3). The process commenced with the preparation of algal cell suspension in BBM medium with the initial concentration of 5-7 billion cells per millimeter. The antialgal effect of various samples was studied on the Mueller Hinton Agarose plates inoculated with algal cultures. The agarose solution $(1 \% w / v)$ of distilled water and bold basal medium (BBM) ( $4: 1$ by volume, respectively) was prepared [19]. The pouring of plates with agar and spreading of algae onto these agar plates was performed in the safety cabinet that was previously sterilized through UV light (10 to 15 minutes) and cleaned with absolute ethanol. The whole process was performed in sterile conditions close to the spirit lamp to avoid any undesired microbial growth except microalgae. Disks of $6 \mathrm{~mm}$ of all samples were placed on agar, and the plates were incubated for 3 to 4 days at $25^{\circ} \mathrm{C}$. The clear zone around sample disks was measured as the growth inhibition diameter of algae [11].

\section{Results and Discussion}

Resultant blends formed by varying the amounts of PEG and SS were characterized by different spectroscopic techniques, and their antifouling potential was ascertaining by antialgal assay. 


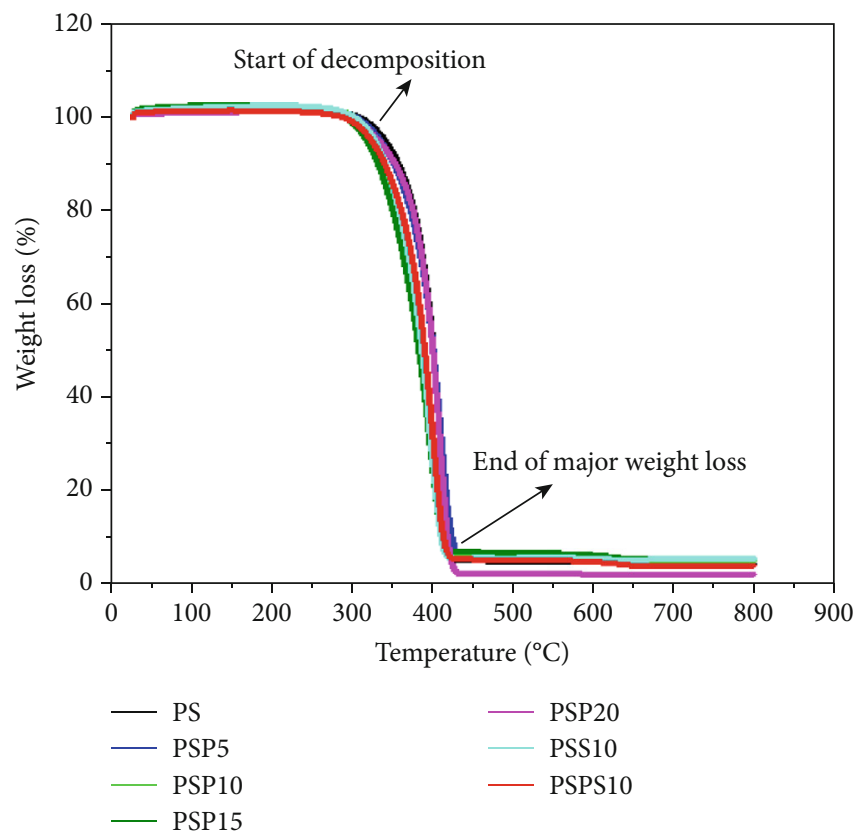

Figure 3: TGA thermographs of the polymer blend samples.

TABle 2: Decomposition temperature $\left(T_{d}\right)$ and melting temperature $\left(T_{m}\right)$ of blends (PS, PSP5-20, PSS10, and PSPS10).

\begin{tabular}{lcc}
\hline Samples & $T_{d}$ & $T_{m}$ \\
\hline PS & 350.3 & 441.2 \\
PSP5 & 316.9 & 431.2 \\
PSP10 & 315.6 & 428.5 \\
PSP15 & 313.4 & 426.7 \\
PSP20 & 310.5 & 425.3 \\
PSS10 & 335.4 & 434.1 \\
PSPS10 & 330.5 & 432.2 \\
\hline
\end{tabular}

3.1. FTIR Spectroscopy of Blends. Various functional groups in the synthesized blends were identified by the FTIR spectroscopy (Figure 2). In the PSS10 blend, the absorption bands at $3060 \mathrm{~cm}^{-1}$ and $3026 \mathrm{~cm}^{-1}$ were due to aromatic C$\mathrm{H}$ stretching vibrations. Three absorption signals at the wave numbers $1600 \mathrm{~cm}^{-1}, 1492 \mathrm{~cm}^{-1}$, and $1452 \mathrm{~cm}^{-1}$ were due to aromatic $\mathrm{C}=\mathrm{C}$ stretching vibrations which has indicated the presence of benzene ring [20]. The absorptions at the $756 \mathrm{~cm}^{-1}$ and $698 \mathrm{~cm}^{-1}$ were corresponded to C-H out-ofplane bending vibrations. This has indicated that there was only one substituent in the benzene ring [21]. SS has exhibited characteristic signals at $1125 \mathrm{~cm}^{-1}$ for $\mathrm{SO}_{2}$ and at $1655 \mathrm{~cm}^{-1}$ for $\mathrm{NH}_{2}$ group which has confirmed the successful blend formation. The IR spectrum of PSP10 blend has shown stretching vibrations of $-\mathrm{OH}$ groups at $3463 \mathrm{~cm}^{-1}$ and $\mathrm{C}-\mathrm{O}$ at $1645 \mathrm{~cm}^{-1}$ for polymeric part corresponding to PEG [22]. In the PSPS10 blend, signatures of all functional group were detected like $\mathrm{C}=\mathrm{C}$ stretching vibrations of $\mathrm{PS}$ at $1452 \mathrm{~cm}^{-1}, \mathrm{C}-\mathrm{O}$ and $-\mathrm{OH}$ signals of PEG at $1645 \mathrm{~cm}^{-1}$ and $3463 \mathrm{~cm}^{-1}$, and $\mathrm{NH}_{2}$ and $\mathrm{SO}_{2}$ bands of SS at $1655 \mathrm{~cm}^{-1}$ and $1125 \mathrm{~cm}^{-1}$, respectively, as shown in Figure 2 .

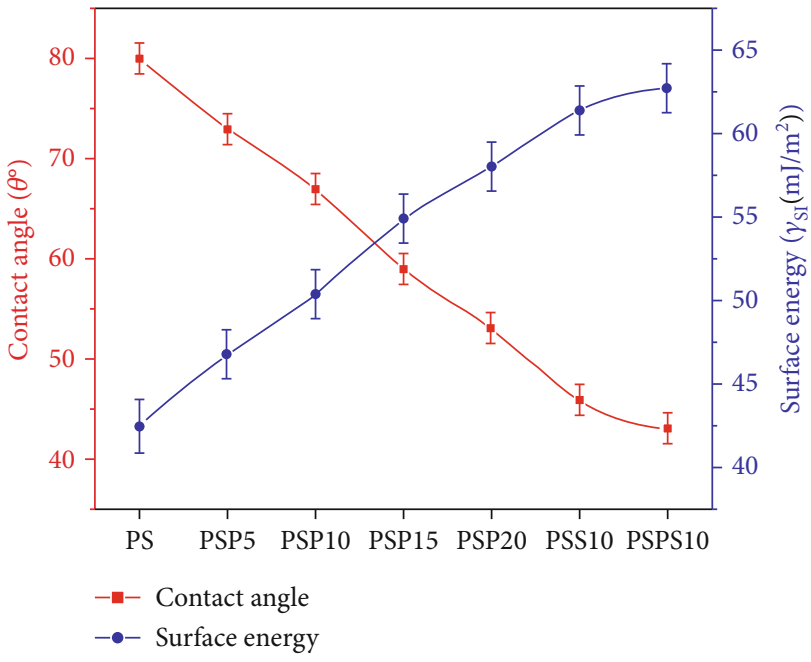

FIgURE 4: Blend composition and water contact angles.

3.2. Thermal Stability. Thermogravimetric analysis (TGA) was performed to determine the thermal stability of polymeric blends [21]. The TGA curves of various polymer blend samples are depicted in Figure 3. The basic thermogravimetric trend corresponding to various samples was almost identical. Thermal decomposition was started in the range of $310^{\circ} \mathrm{C}$ to $350^{\circ} \mathrm{C}$ as given in Table 2 . The major structural decomposition occurred between $350^{\circ} \mathrm{C}$ and $420^{\circ} \mathrm{C}$ with the negligible amount of decomposition and continued till $800^{\circ} \mathrm{C}$. Blending has affected the thermal stability of PS which depends upon the amount of PEG incorporated into blends, although it tends to decrease the thermal stability of polystyrene blends but not more than $25^{\circ} \mathrm{C}$ at any decomposition stage [21]. PEG is relatively a low-melting polymer in comparison with polystyrene that has reduced the thermal stability of corresponding blends while the initial 


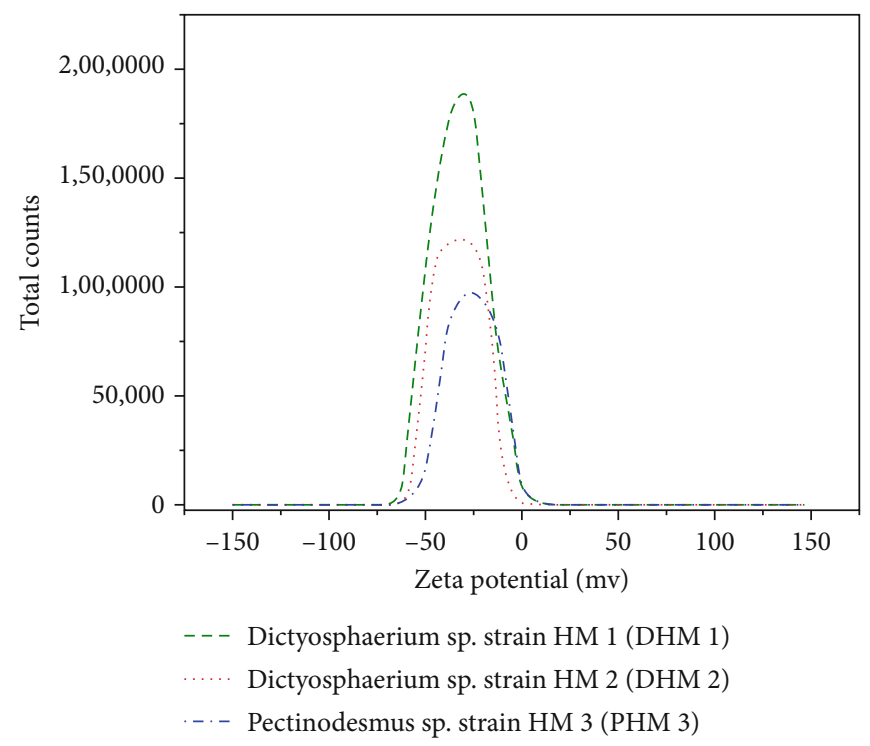

FIgure 5: Zeta Potential of algal strains DHM1, DHM2, and DHM3.

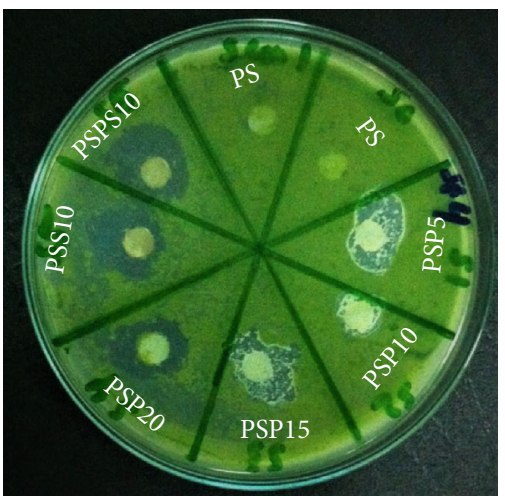

(a)



(b)

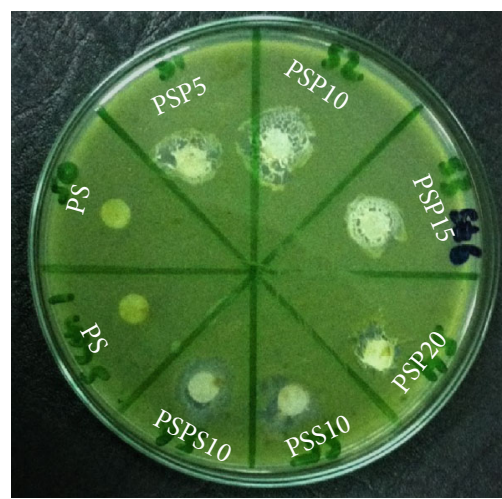

(c)

Figure 6: Microalgae-cultured plates with the disk diffusion method against (a) Dictyosphaerium sp., strain HM1 (DHM1), (b) Dictyosphaerium sp., strain HM2 (DHM2), and (c) Pectinodesmus sp., strain HM3 (PHM3).

decomposition temperature of blends remains high in comparison with room temperature where most of the polymers are practically used [21].

Contrary to PEG, SS has improved the thermal stability of PSS10 polymer blends to a notable extent. Ternary blend PSPS10 tends to decompose by few degrees earlier when compared with binary PSS10 blends in most of the cases due to PEG. All polymeric blends were found thermally stable even at $300^{\circ} \mathrm{C}[23,24]$.

3.3. Contact Angle and Surface Energy. Hydrophilicity of various polymer blends was measured in terms of contact angle as shown in Figure 4. The static contact angle of virgin PS was found to be $80^{\circ}$, and increasing the concentration of PEG, the contact angle was decreased [25]. The PSP5 blend with 5\% PEG concentration has demonstrated contact angle of $66^{\circ}$ [26]. By increasing the concentration of PEG up to $10 \%$ in the PSP 10 blend, the contact angle was found to be $63^{\circ}$ [27]. The PSP15 and PSP20 blends with 15\% and $20 \%$ concentration of PEG shown to have the contact angles of $65^{\circ}$ and $53^{\circ}$, respectively [27]. Since PEG is hydrophilic in nature due to the presence of hydroxyl group, therefore by increasing the PEG contents, it has increased hydrophilicity of the blends which has resulted in the decrease of water contact angle $[28,29]$.

Further addition of $10 \%$ silver sulfadiazine (SS) in the PSS10 blend has increased the hydrophilicity due to the polar nature of SS and decreased the contact angle of blend to $46^{\circ}$ [22]. The polarity of SS has caused more strong attraction of water molecules on blend's surface [30]. The PSPS10 blend with $10 \%$ SS and 10\% PEG concentration has shown the lowest contact angle, i.e., $43^{\circ}$. The decrease in the contact angle of PSPS10 is due to presence of hydroxyl group in PEG and polarity of SS that tends to increase the hydrophilic nature of PSP10 [31, 32].

Among various blend compositions, the surface energy $(\gamma s)$ of virgin PS has exhibited the minimum surface energy $\left(42 \pm 1 \mathrm{~mJ} / \mathrm{m}^{2}\right)$, and its surface energy has increased from 47 to $58 \mathrm{~mJ} / \mathrm{m}^{2}$ upon addition of $5-20 \%$ hydrophilic PEG in PS, respectively [33]. Upon addition of SS into PS, it caused 
further increased of surface energy up to $61 \mathrm{~mJ} / \mathrm{m}^{2}$ in the PSS10 blend while the PSPS10 ternary blend has exhibited the maximum $\gamma s$ value, i.e., $63 \mathrm{~mJ} / \mathrm{m}^{2}$ [34]. Surface energy and contact angle have inverse relationship as shown in Figure 4. Generally, higher surface energy of material caused more bioadhesion and reduced antialgal activity [35]. But in this study, increase of surface energy did not lead to decrease of antialgal activities. This has indicated that biofilm adhesion is not a major antialgal factor in these blended materials.

3.4. Zeta Potential and Size of Algal Strains. Zeta potential and surface charges are important features that determine surface properties like hydrophilicity, hydrophobicity, and bioadhesion [36, 37]. Zeta potential values of Dictyosphaerium sp. (DHM1), Dictyosphaerium sp. (DHM2), and Pectinodesmus sp. (PHM3) algal strains were found to be $-32.7 \mathrm{mV},-33.0 \mathrm{mV}$, and $-25.7 \mathrm{mV}$, respectively (Figure 5) [38], whereas the average diameter of algal strains was measured as $179.6 \mathrm{~nm}, 102.6 \mathrm{~nm}$, and $70.4 \mathrm{~nm}$ corresponding to Dictyosphaerium sp. (DHM1), Dictyosphaerium sp. (DHM2), and Pectinodesmus sp. (PHM3), respectively. There was no discernible difference in the zeta potential of different algae strains. Therefore, all the strains have exhibited almost identical behavior against different polymer blends. Microbial organisms are negatively charged over a wide $\mathrm{pH}$ range [39]. Zeta potential leads to electrostatic interaction that results in the adhesion of microorganisms on surfaces; thus, fouling may increase with the increase in zeta potential [40].

3.5. Antialgal Activity. The inhibition of algal growth by polymer blends was quantitatively measured in terms of inhibition zone diameter [11]. The inhibition zones of polymer blends against Dictyosphaerium sp. (DHM1), Dictyosphaerium sp. (DHM2), and Dictyosphaerium sp. (DHM3) are depicted in Figures 6 and 7. The green lawn on agar plates has confirmed algal growth while each tested polymer blend has exhibited particular inhibition zone depending upon its composition and concentration [41]. Results have revealed that PS has demonstrated no inhibition zone thus no antifouling activity. Among various PSP5-20 blends, PSP5 has the lowest PEG, and PSP20 has the highest PEG concentration. From PSP5 to PSP20, the inhibition zone against DHM1 was increased from $8.5 \mathrm{~mm}$ to $9.3 \mathrm{~mm}$, against DHM2 from $9.1 \mathrm{~mm}$ to $9.3 \mathrm{~mm}$, and against DHM3 from $8.6 \mathrm{~mm}$ to $9.7 \mathrm{~mm}$, respectively [42]. This has clearly shown the direct relationship between the amount of hydrophilic PEG in blend and its antialgal properties [43]. The inhibition zone was increased with the addition of SS in PSS10 blend $10.2 \mathrm{~mm}, 10.2 \mathrm{~mm}$, and $11.4 \mathrm{~mm}$ inhibition zone against DHM1, DHM2, and DHM3 algal strains, respectively, as given in Table 3. Most significant antialgal behavior was observed in the PSPS10 blend owing to the presence of both hydrophilic components, i.e., PEG and SS. This has demonstrated the highest zone of inhibition, i.e., $10.5 \mathrm{~mm}, 10.5 \mathrm{~mm}$, and $11.4 \mathrm{~mm}$ against DHM1, DHM2, and DHM3 algal strains, respectively, as depicted in Figures 6 and 7.

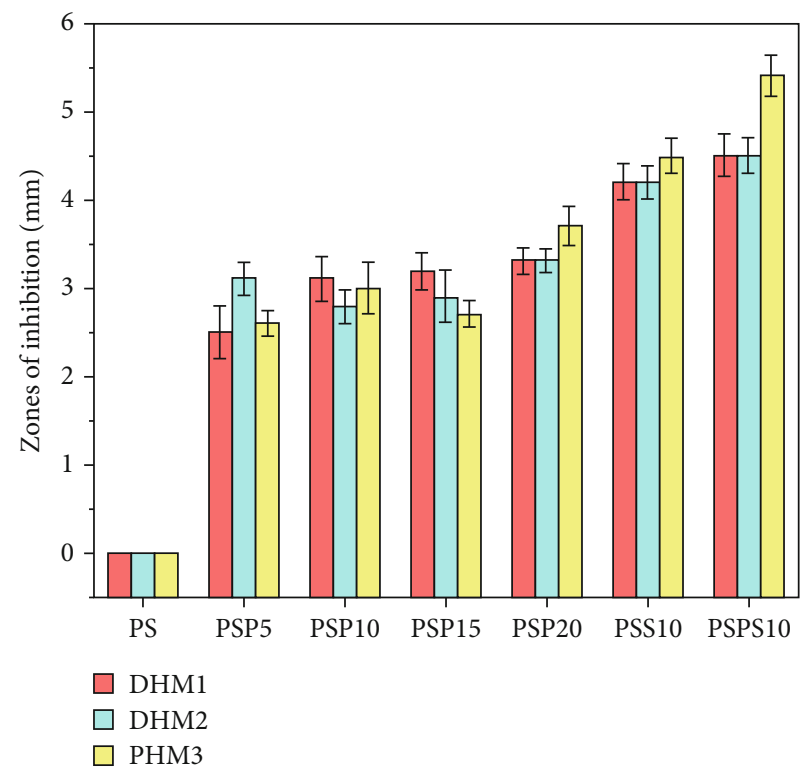

Figure 7: The antialgal analysis of various compositions of blends (PS, PSP5-20, PSS10, and PSPS10) against different algal strains (DHM1, DHM2, and PHM3).

TABLE 3: Contact angle, surface energy, and zone of inhibition of blends (PS, PSP5-20, PSS10, and PSPS10).

\begin{tabular}{lccccc}
\hline \multirow{2}{*}{ Samples } & Contact & Surface & \multicolumn{3}{c}{ Zone of inhibition $(\mathrm{mm})$} \\
& angle $(\theta)$ & energy $(\gamma s)$ & DHM1 & DHM2 & PHM3 \\
\hline PS & 80 & 42.52 & 0 & 0 & 0 \\
PSP5 & 73 & 46.83 & 8.5 & 9.1 & 8.6 \\
PSP10 & 67 & 50.42 & 9.1 & 8.8 & 9 \\
PSP15 & 59 & 54.92 & 9.2 & 8.9 & 8.7 \\
PSP20 & 53 & 58.07 & 9.3 & 9.3 & 9.7 \\
PSS10 & 46 & 61.41 & 10.2 & 10.2 & 10.5 \\
PSPS10 & 43 & 62.74 & 10.5 & 10.5 & 11.4 \\
\hline
\end{tabular}

The hydrophilicity, high mobility, conformation, neutral charge, and hydrogen bonding capacities of PEG are considered critical in their antifouling efficiency [44]. The hydrophilic character of PEG lowers the interfacial free energy for the water thus weakens the adsorption forces for proteins [45]. When a protein reaches the PEG mount surface, then a limited amount of conformation would be available in the PEG chains due to steric stabilization [46]. The protein will concurrently displace any water connected to PEG units, thus altering the state of water polymer solvation [47]. Both phenomenon, i.e., hydrophilicity and conformation would boost the system's free energy, so the mechanism would not be conducive, and the protein would retain away from the surface $[47,48]$. The high mobility of PEG chains would shorten the protein interaction time on the surface, thereby minimizing the adsorbed volumes [49]. Moreover, hydrogen bonding of water molecules across the PEG chains produces some sort of bound water shell structure that serves to deter proteins from approaching $[50,51]$. 
Incorporation of SS to blends PSS10 and PSPS10 resulted in even higher algal growth inhibition in comparison with PSP5-20. The amino group present in SS has enhanced the antialgal activity of materials because it increased the hydrophilic nature of blends [52]. Owing to the hydrophilicity of SS, hydration shells of water are formed on the blend surface that repels algal strains [53]. The ternary blend PSPS10 was proved to be the best prepared composition that resulted in the maximum algal growth inhibition of $10.5 \mathrm{~mm}$ for DHM1, $10.5 \mathrm{~mm}$ for DMH2, and $11.4 \mathrm{~mm}$ for PHM3 due to the presence of strong antifouling agent silver sulfadiazine and PEG.

\section{Conclusions}

The antialgal synergistic potential of polystyrene blends with polyethylene glycol and silver sulfadiazine is revealed in this work. Results have concluded that both antialgal agents, i.e., PEG and SS, have worked synergistically in polystyrene matrix to improve the antialgal potential as evident from a direct relationship between the amount of antialgal agent and its inhibition zones against microbial growth. All prepared compositions of PSP, PSS, and PSPS blends were proved quite effective against algal species Dictyosphaerium sp. (DHM1), Dictyosphaerium sp. (DHM2), and Pectinodesmus sp. (PHM3) while the ternary blend PSPS10 was the most effective one due to the cooccurrence of both antialgal agents and exhibited maximum zone of inhibitions against all algal strains. Contact angle and surface energy values have highlighted that bioadhesion is not a limiting factor in the fouling of these prepared blends. Thermographs obtained from TGA have demonstrated the thermal stability of blends while a zetasizer has measured the significant values of zeta potential and particle size. These significant results about thermal stability and antialgal features of prepared polystyrene/ polyethylene glycol/silver sulfadiazine blends have widen their scope in the fields of marine coatings.

\section{Data Availability}

The data used to support the findings of this study is included within the article.

\section{Conflicts of Interest}

The authors declare no conflict of interest.

\section{Acknowledgments}

All authors are grateful to the NUST Research Directorate for financial assistance. Dr. Nasir M. Ahmad acknowledge HEC, NRPU through Project No. 3526 and 6020, for financial support.

\section{References}

[1] C. C. C. R. de Carvalho, "Marine biofilms: a successful microbial strategy with economic implications," Frontiers in Marine Science, vol. 5, pp. 1-11, 2018.
[2] G. D. Bixler and B. Bhushan, "Biofouling: lessons from nature," in Philosophical Transactions of the Royal Society A: Mathematical, Physical and Engineering Sciences, vol. 370, no. 1967, 2012.

[3] A. S. Carlini, L. Adamiak, and N. C. Gianneschi, "Biosynthetic Polymers as Functional Materials," Macromolecules, vol. 49, no. 12, pp. 4379-4394, 2016.

[4] M. Salta, J. A. Wharton, P. Stoodley et al., "Designing biomimetic antifouling surfaces," Philosophical Transactions of the Royal Society A: Mathematical, Physical and Engineering Sciences, vol. 368, pp. 4729-4754, 2010.

[5] M. Cobos, I. De-La-Pinta, G. Quindós, M. J. Fernández, and M. D. Fernández, "Synthesis, physical, mechanical and antibacterial properties of nanocomposites based on poly (vinyl alcohol)/graphene oxide-silver nanoparticles," Polymers, vol. 12, pp. 1-18, 2020.

[6] Z. Qiao, D. Xu, Y. Yao, S. Song, M. Yin, and J. Luo, "Synthesis and antifouling activities of fluorinated polyurethanes," Polymer International, vol. 68, pp. 1361-1366, 2019.

[7] S. Aishwarya, J. Shanthi, and R. Swathi, "Surface energy calculation using Hamaker's constant for polymer/silane hydrophobic thin films," Materials Letters, vol. 253, pp. 409-411, 2019.

[8] A. M. C. Maan, A. H. Hofman, W. M. de Vos, and M. Kamperman, "Recent developments and practical feasibility of polymer-based antifouling coatings," Advanced Functional Materials, vol. 30, 2020.

[9] B. Ran, C. Jing, C. Yang, X. Li, and Y. Li, "Synthesis of efficient bacterial adhesion-resistant coatings by one-step polydopamine-assisted deposition of branched polyethylenimine-g-poly(sulfobetaine methacrylate) copolymers," Applied Surface Science, vol. 450, pp. 77-84, 2018.

[10] A. Krywko-Cendrowska, S. di Leone, M. Bina, S. YorulmazAvsar, C. G. Palivan, and W. Meier, "Recent advances in hybrid biomimetic polymer-based films: from assembly to applications," Polymers, vol. 12, 2020.

[11] A. Gitchaiwat, A. Kositchaiyong, K. Sombatsompop, B. Prapagdee, K. Isarangkura, and N. Sombatsompop, “Assessment and characterization of antifungal and antialgal performances for biocide-enhanced linear low-density polyethylene," Journal of Applied Polymer Science, vol. 128, pp. 371-379, 2013.

[12] W. Yandi, S. Mieszkin, M. E. Callow et al., "Antialgal activity of poly(2-(dimethylamino)ethyl methacrylate) (PDMAEMA) brushes against the marine alga Ulva," Biofouling, vol. 33, pp. 169-183, 2017.

[13] R. B. Bodkhe, S. J. Stafslien, J. Daniels et al., "Zwitterionic siloxane-polyurethane fouling-release coatings," Progress in Organic Coatings, vol. 78, 2014.

[14] S. Mushtaq, N. M. Ahmad, H. Nasir, A. Mahmood, and H. A. Janjua, "Transpicuous-cum-fouling resistant copolymers of 3sulfopropyl methacrylate and methyl methacrylate for optronics applications in aquatic medium and healthcare," Advances in Polymer Technology, vol. 2020, 11 pages, 2020.

[15] J. S. Suk, Q. Xu, N. Kim, J. Hanes, and L. M. Ensign, "PEGylation as a strategy for improving nanoparticle-based drug and gene delivery," Advanced Drug Delivery Reviews, vol. 99, pp. 28-51, 2016.

[16] J. Wu, W. Lin, Z. Wang, S. Chen, and Y. Chang, "Investigation of the hydration of nonfouling material poly(sulfobetaine methacrylate) by low-field nuclear magnetic resonance," Langmuir, vol. 28, pp. 7436-7441, 2012. 
[17] B. K. D. Ngo and M. A. Grunlan, "Protein resistant polymeric biomaterials," ACS Macro Letters, vol. 6, pp. 992-1000, 2017.

[18] S. R. Meyers and M. W. Grinstaff, "Biocompatible and bioactive surface modifications for prolonged in vivo efficacy," Chemical Reviews, vol. 112, pp. 1615-1632, 2012.

[19] S. Newport, B. Differ, K. Hoelzer et al., "Agar disk diffusion and automated microbroth dilution produce similar antimicrobial susceptibility testing results," Foodborne pathogens and disease, vol. 8, 2011.

[20] F. Junfei, X. Yimin, and L. I. Qiang, "Preparation of polystyrene spheres in different particle sizes and assembly of the PS colloidal crystals," Science China Technological Sciences, vol. 53, pp. 3088-3093, 2010.

[21] A. Sari, C. Alkan, and A. Biçer, "Synthesis and thermal properties of polystyrene-graft-PEG copolymers as new kinds of solid-solid phase change materials for thermal energy storage," Materials Chemistry and Physics, vol. 133, pp. 87-94, 2012.

[22] X. Liu, H. Gan, C. Hu et al., "Silver sulfadiazine nanosuspension-loaded thermosensitive hydrogel as a topical antibacterial agent," International Journal of Nanomedicine, vol. 14, pp. 289-300, 2019.

[23] H. Bley, B. Fussnegger, and R. Bodmeier, "Characterization and stability of solid dispersions based on PEG/polymer blends," International Journal of Pharmaceutics, vol. 390, pp. 165-173, 2010.

[24] F. H. Falqi, O. A. Bin-Dahman, M. Hussain, and M. A. AlHarthi, "Preparation of Miscible PVA/PEG Blends and Effect of Graphene Concentration on Thermal, Crystallization, Morphological, and Mechanical Properties of PVA/PEG (10 wt\%) Blend," International Journal of Polymer Science, vol. 2018, 10 pages, 2018.

[25] G. Lin, X. Zhang, S. R. Kumar, and J. E. Mark, "Improved hydrophilicity from poly(ethylene glycol) in amphiphilic conetworks with poly(dimethylsiloxane)," Silicon, vol. 1, pp. 173181, 2009.

[26] M. Inutsuka, H. Tanoue, N. L. Yamada, K. Ito, and H. Yokoyama, "Dynamic contact angle on a reconstructive polymer surface by segregation," RSC Advances, vol. 7, pp. 17202-17207, 2017.

[27] L. Li, S. Liu, R. Liu, C. Geng, and Z. Hu, "Preparation and characterization of hydrophilic wetting-modified polyamide fibers," Advances in Polymer Technology, vol. 2020, 7 pages, 2020.

[28] Y. Ma, F. Shi, J. Ma, M. Wu, J. Zhang, and C. Gao, "Effect of PEG additive on the morphology and performance of polysulfone ultrafiltration membranes," Desalination, vol. 272, pp. 51-58, 2011.

[29] M. A. Abbas, S. Mushtaq, W. A. Cheema et al., "Surface Modification of TFC-PA RO Membrane by Grafting Hydrophilic pH Switchable Poly(Acrylic Acid) Brushes," Advances in Polymer Technology, vol. 2020, 12 pages, 2020.

[30] H. Razavi, M. H. Darvishi, and S. Janfaza, "Silver sulfadiazine encapsulated in lipid-based nanocarriers for burn treatment," Journal of Burn Care \& Research, vol. 39, pp. 319-325, 2018.

[31] P. Kim, D. H. Kim, B. Kim et al., "Fabrication of nanostructures of polyethylene glycol for applications to protein adsorption and cell adhesion," Nanotechnology, vol. 16, pp. 24202426, 2005.

[32] A. Taglietti, G. Dacarro, D. Barbieri et al., "High bactericidal self-assembled nano-monolayer of silver sulfadiazine on hydroxylated material surfaces," Materials, vol. 12, 2019.
[33] Y. C. Chao, S. K. Su, Y. W. Lin, W. T. Hsu, and K. S. Huang, "Interfacial properties of polyethylene glycol/vinyltriethoxysilane (PEG/VTES) copolymers and their application to stain resistance," Journal of Surfactants and Detergents, vol. 15, pp. 299-305, 2012.

[34] M. Venkataraman and M. Nagarsenker, "Silver sulfadiazine nanosystems for burn therapy," AAPS PharmSciTech, vol. 14, pp. 254-264, 2013.

[35] C. A. Del Grosso, C. Leng, K. Zhang et al., "Surface hydration for antifouling and bio-adhesion," Chemical Science, vol. 11, no. 38, pp. 10367-10377, 2020.

[36] J. A. Finlay, M. E. Callow, L. K. Ista, G. P. Lopez, and J. A. Callow, "The influence of surface wettability on the adhesion strength of settled spores of the green alga Enteromorpha and the diatom amphora," Integrative and Comparative Biology, vol. 42, pp. 1116-1122, 2002.

[37] F. Hejda, P. Sola", and J. Kousal, "Surface free energy determination by contact angle measurements - a comparison of various approaches," WDS'10, pp. 25-30, 2010.

[38] M. Khalid, N. Khalid, I. Ahmed, R. Hanif, M. Ismail, and H. A. Janjua, "Comparative studies of three novel freshwater microalgae strains for synthesis of silver nanoparticles: insights of characterization, antibacterial, cytotoxicity and antiviral activities," Journal of Applied Phycology, vol. 29, pp. 1851-1863, 2017.

[39] W. F. Walkenhorst, J. W. Klein, P. Vo, and W. C. Wimley, "PH dependence of microbe sterilization by cationic antimicrobial peptides," Antimicrobial Agents and Chemotherapy, vol. 57, pp. 3312-3320, 2013.

[40] O. Nedela, P. Slepicka, and V. Švorcík, "Surface modification of polymer substrates for biomedical applications," Materials, vol. 10, no. 10, p. 1115, 2017.

[41] D. Debalke, M. Birhan, A. Kinubeh, and M. Yayeh, “Assessments of antibacterial effects of aqueous-ethanolic extracts of Sida rhombifolia's aerial part," Scientific World Journal, vol. 2018, pp. 1-8, 2018.

[42] K. M. Dobosz, C. A. Kuo-Leblanc, T. Emrick, and J. D. Schiffman, "Antifouling ultrafiltration membranes with retained pore size by controlled deposition of Zwitterionic polymers and poly(ethylene glycol)," Langmuir, vol. 35, pp. 1872-1881, 2019.

[43] T. Ekblad, G. Bergström, T. Ederth et al., "Poly(ethylene glycol)-containing hydrogel surfaces for antifouling applications in marine and freshwater environments," Biomacromolecules, vol. 9, no. 10, pp. 2775-2783, 2008.

[44] S. Chen, L. Li, C. Zhao, and J. Zheng, "Surface hydration: principles and applications toward low-fouling/nonfouling biomaterials," Polymer, vol. 51, pp. 5283-5293, 2010.

[45] C. Sanchez-Cano and M. Carril, "Recent developments in the design of non-biofouling coatings for nanoparticles and surfaces," International Journal of Molecular Sciences, vol. 21, pp. 1-24, 2020.

[46] P. B. Lawrence and J. L. Price, "How PEGylation influences protein conformational stability," Current Opinion in Chemical Biology, vol. 34, pp. 88-94, 2016.

[47] L. Guerrini, R. A. Alvarez-Puebla, and N. Pazos-Perez, "Surface modifications of nanoparticles for stability in biological fluids," Materials, vol. 11, pp. 1-28, 2018.

[48] K. Terpiłowski, "Apparent surface free energy of polymer/ paper composite material treated by air plasma," International Journal of Polymer Science, vol. 2017, 8 pages, 2017. 
[49] A. Larsson, T. Ekblad, O. Andersson, and B. Liedberg, "Photografted poly(ethylene glycol) matrix for affinity interaction studies," Biomacromolecules, vol. 8, pp. 287-295, 2007.

[50] C. Q. Sun, "Aqueous charge injection: solvation bonding dynamics, molecular nonbond interactions, and extraordinary solute capabilities," International Reviews in Physical Chemistry, vol. 37, pp. 363-558, 2018.

[51] A. C. Noguer, S. Kiil, and S. Hvilsted, "Long-Term Stability of PEG-Based Antifouling Surfaces in a Marine Environment," in 12th Coatings Science International Conference 2016, Noordwijk, Netherlands, 2016.

[52] T. Dai, Y.-Y. Huang, K. S. Sharma, T. J. Hashmi, B. D. Kurup, and R. M. Hamblin, "Topical antimicrobials for burn wound infections," Recent patents on anti-infective drug discovery, vol. 5, no. 2, pp. 124-151, 2010.

[53] I. Francolini, C. Vuotto, A. Piozzi, and G. Donelli, "Antifouling and antimicrobial biomaterials: an overview," Apmis, vol. 125, pp. 392-417, 2017. 